\title{
CONSIDERATION OF THE MICROSTRUCTURE OF PARTICLE SUSPENSION TO ESTIMATE ITS INTRINSIC VISCOSITY
}

\author{
TOMOHIRO FUKUI, MISA KAWAGUCHI \& KOJI MORINISHI \\ Department of Mechanical Engineering, Kyoto Institute of Technology, Japan
}

\begin{abstract}
It is important to comprehend rheology of particle suspension to facilitate useful and effective applications in many fields. It is well known that relative viscosity for higher concentration becomes higher than that form Einstein's viscosity equation. One of the reasons is interaction between suspended particles and suspending fluids. We previously considered the influence of interactions on increase in relative viscosity by focusing on the suspended particles' rotational motions. For higher concentrated suspensions, the rotational motions were disturbed because particles were too jammed to move freely, especially in rotational direction. Since these rotational motions were strongly related to the total macroscopic fluid resistance, the relative viscosity depended on the microscopic gaps between the particles. In the meantime, relationships between microstructure, i.e., spatial arrangement of the particles, and rheology are still unclear. In this study, therefore, numerical simulations were conducted to consider relative and intrinsic viscosities in terms of microstructure of suspensions. The results showed that the concentration profile of the particle suspension was almost flat except for near the channel wall. Few particles flowed near the wall because repulsive force from the wall increased exponentially with approaching the wall. They flowed away from the channel wall. For the higher concentrated suspension, on the other hand, particles were too jammed to flow smoothly near the channel center. Then some particles were pushed out toward the channel wall against the repulsive force. In this case, they flowed near the channel wall as well. Owing to these effects, the concentration profile for the concentrated suspension depicted almost flat including near the wall. The microstructure for higher concentrated suspension also changed with changes in concentration profile. Then the relative and intrinsic viscosities were consequently increased with concentration. The intrinsic viscosity was significantly related to the microstructure of the suspension.

Keywords: rheology, non-Newtonian property, dilute suspension, microstructure, margination, twoway coupling simulation.
\end{abstract}

\section{INTRODUCTION}

It is important to comprehend rheology of particle suspension to facilitate useful and effective applications in many fields. One of the most convenient aspects by using particle suspension is its simple relationship between particle concentration and consequent apparent viscosity. According to Einstein's viscosity equation [1], the apparent effective viscosity $\eta_{\text {eff }}$ can be simply related to the particle concentration $\phi$ with intrinsic viscosity $[\eta]$ :

$$
\eta_{\mathrm{eff}}=\eta_{0}\{1+[\eta] \phi\}
$$

where $\eta_{0}$ is the viscosity of the solvent. The intrinsic viscosity [ $\left.\eta\right]$ depends on the shape of the suspended particles: for the case of spherical particles, $[\eta]=2.0$ for two-dimensional [2] and $[\eta]=2.5$ for three-dimensional [1]. In order to compare contributions of suspended particles to the apparent viscosity among suspensions with different solvent, relative viscosity $\eta_{\text {eff }} / \eta_{0}$ is sometimes preferably employed: 


$$
\frac{\eta_{\mathrm{eff}}}{\eta_{0}}=1+[\eta] \phi
$$

It is well known that relative viscosity for higher concentration becomes higher than that form Einstein's viscosity equation. One of the reasons is interaction between suspended particles and suspending fluids [3]-[6]. We previously considered the influence of interactions on increase in relative viscosity by focusing on the suspended particles' rotational motions [7], [8]. For higher concentrated suspensions, the rotational motions were disturbed because particles were too jammed to move freely, especially in rotational direction. Since these rotational motions were strongly related to the total macroscopic fluid resistance [7], the relative viscosity, i.e., pressure loss mainly due to viscous dissipation, depended on the microscopic gaps between the particles.

Recently, effects of non-Newtonian properties of the solvent have also received much attention. In a non-Newtonian solvent, since macroscopic velocity profiles strongly depend on the shear rate, suspended particles' behaviors are entirely different from those in a Newtonian solvent. For example, Hu et al. [9] and Christ et al. [10] showed the preferable radial equilibrium positions for the suspended particles in non-Newtonian fluids. Tanaka et al. [11] reported effects of the power-law fluidic properties on the suspension rheology. They successfully showed increase in relative and intrinsic viscosities of a suspension attributed by the non-Newtonian solvent. These findings are important especially in a field of bioengineering. It is reported that blood from patients suffering from cardiovascular disease included much more proteins within the plasma [12]. This may lead to higher viscosities of blood. They also showed higher death rate related to large amount of proteins in plasma. It is important to consider mechanisms of viscosity changes due to interactions between suspending fluid and suspended particles.

In the meantime, relationships between microstructure, i.e., spatial arrangement of the particles [13], and rheology are still unclear. Doyeux et al. [6] considered effects of particle's radial position on the total effective viscosity. They showed that when a particle approached the channel wall, the effective viscosity increased exponentially. Thus, the effective viscosity is not only a function of concentration $\phi$ but also strongly influenced by its microstructure. They also proposed an alternative estimation for effective viscosity considering its microstructure instead of Einstein's equation. Based on their proposal, Okamura et al. [14] recently validated total relative viscosity of a suspension by considering summation of each particle's contribution. They showed that relative viscosity could be estimated by its microstructure in a limited condition. Although their study was still preliminary, viscosity estimation by its microstructure would be a promising approach and more considerations should be necessary. It is also expected to reveal the mechanism of viscosity changes by considering microstructure of a suspension. Fukui et al. [15] showed microstructure changes due to inertial effects of the suspended particles and consequent viscosity decrease of suspension. These microstructure changes were considered to be one of the major factors in changing macroscopic rheology. On the other hand, since these inertial effects generally tended to cause thixotropic behavior, i.e., viscosity decrease, it is important to consider microstructure changes resulting in viscosity increase as discussed above. In this study, we focus on the microstructure in a pressure-driven suspension flow with different concentrations in order to consider mechanism of viscosity increase with increasing concentration. We also consider the relationship between microstructure and its relative and intrinsic viscosities. 


\section{METHODS}

\subsection{Computational models}

We conducted two-dimensional pressure-driven suspension flow simulations by a two-way coupling scheme. Fig. 1 shows a schematic view of the simulation model used in this study. The cannel width was $2 l=400 \mu \mathrm{m}$, and the axial length was set 4 times as long as its width. A periodic boundary condition considering pressure was applied axial direction to reduce computational costs. Suspended particles with a diameter $2 r=20 \mu \mathrm{m}$ were randomly distributed as an initial condition. Note that at least $20 \mu \mathrm{m}$ gaps between the particles or particle-wall were allowed for the initial position to stabilize the computation. Number of particles was set 21 for $\phi=1.02 \%, 42$ for $\phi=2.04 \%$, and 84 for $\phi=4.07 \%$, respectively. The simulations were then conducted until physical time $t=8 \mathrm{~s}$, which corresponds to nondimensional time of 100 . More or less, initial random positions of the particles affect particles flow patterns and consequent microstructure [15], these simulations were repetitively carried out 40 times for $\phi=1.02 \%$, and 20 times for $\phi=2.04$ and $4.07 \%$, respectively. The spatial resolution was set $1 \mu \mathrm{m}$ for both directions, which has been validated by grid independence test [8]. Particle shape was described by virtual flux method [16] to satisfy the hydrodynamic boundary conditions on the particle surface in Cartesian coordinate system with regular intervals.

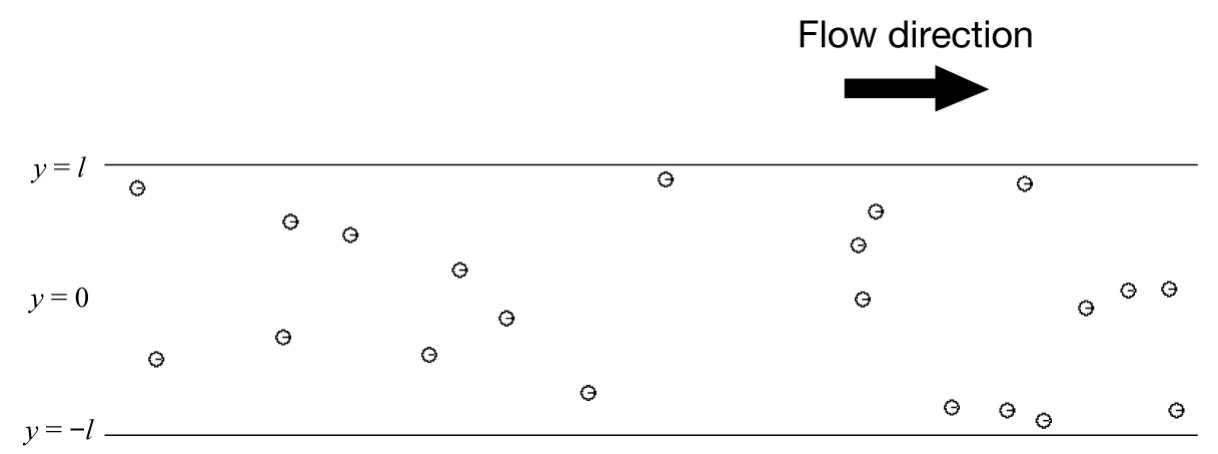

Figure 1: Schematic view of a pressure-driven suspension flow model. The channel width and length were set $400 \mu \mathrm{m}$ and $1,600 \mu \mathrm{m}$, respectively. Periodic boundary condition was applied in the $x$ direction. Suspended particles with a diameter $2 r=20 \mu \mathrm{m}$ were randomly distributed as an initial condition. Number of particles was set 21 for $\phi=1.02 \%, 42$ for $\phi=2.04 \%$, and 84 for $\phi=4.07 \%$, respectively.

\subsection{Governing equation for suspending fluid}

The governing equation for suspending fluid was regularized lattice Boltzmann equation [17], [18], which is a modified form of the original lattice Boltzmann equation in order to stabilize the computation. Briefly, distribution function $f_{\alpha}$ in the regularized lattice Boltzmann equation is written by using up to second-order moments:

$$
f_{\alpha}=\omega_{\alpha}\left(a_{0}+b_{i} e_{\alpha i}+c_{i j} e_{\alpha i} e_{\alpha j}\right)
$$


where $\omega$ is the weight factor, $\alpha$ is the direction of the discrete velocity vector $\boldsymbol{e}_{\alpha}$, and $a_{0}, b_{i}$, $c_{i j}$ are the parameters that satisfy the following relationships:

$$
\begin{gathered}
\sum_{\alpha} f_{\alpha}=\rho, \\
\sum_{\alpha} e_{\alpha i} f_{\alpha}=\rho u_{i} \\
\sum_{\alpha} e_{\alpha i} e_{\alpha j} f_{\alpha}=\frac{c^{2}}{3} \rho \delta_{i j}+\rho u_{i} u_{j}+\Pi_{i j}^{\mathrm{neq}},
\end{gathered}
$$

where $\Pi_{i j}{ }^{\text {neq }}$ is the nonequilibrium part of the stress tensor. The distribution function $f_{a}$ in eqn (3) is then,

$$
f_{\alpha}=\omega_{\alpha} \rho\left[1+\frac{3\left(e_{\alpha i} u_{i}\right)}{c^{2}}+\frac{9\left(e_{\alpha i} u_{i}\right)^{2}}{2 c^{4}}-\frac{3\left(u_{i} u_{i}\right)}{2 c^{2}}\right]+\frac{9 \omega_{\alpha}}{2 c^{2}}\left(\frac{e_{\alpha i} e_{\alpha j}}{c^{2}}-\frac{1}{3} \delta_{i j}\right) \Pi_{i j}^{\mathrm{neq}} .
$$

The first term is equivalent to Maxwell equilibrium distribution function $f_{\alpha}^{\text {eq }}$ with low Mach number approximation. When the distribution function $f_{\alpha}$ can be expanded by a power series of Kundsen number $\varepsilon$ around the equilibrium distribution function $f_{\alpha}^{\text {eq }}$, the distribution function $f_{\alpha}$ is written as

$$
f_{\alpha}=f_{\alpha}^{\mathrm{eq}}+f_{\alpha}^{\mathrm{neq}}=f_{\alpha}^{0}+f_{\alpha}^{1}+f_{\alpha}^{2}+\cdots
$$

where $f_{\alpha}^{n}$ corresponds to of the order of $O\left(\varepsilon^{n}\right)$, and $f_{\alpha}{ }^{0}$ is equal to $f_{\alpha}^{\text {eq }}$. Therefore, the second term of eqn (7) can be substituted for $f_{\alpha}{ }^{1}$, and the time evolution equation for the regularized lattice Boltzmann equation is

$$
f_{\alpha}\left(t+\Delta t, \boldsymbol{x}+\boldsymbol{e}_{\alpha} \Delta t\right)=f_{\alpha}^{\mathrm{eq}}(t, \boldsymbol{x})+\left(1-\frac{1}{\tau}\right) f_{\alpha}^{1}(t, \boldsymbol{x})
$$

where $\tau$ is the relaxation time. When Navier-Stokes equations are derived from lattice Boltzmann equation through Chapman-Enskog expansion procedure, the relaxation time $\tau$ is defined as follows in the incompressible limit [19],

$$
\tau=\frac{3 v}{c \Delta x}+\frac{\Delta t}{2}
$$

where $v$ is the kinematic viscosity. The relaxation time $\tau=0.74$ for all our computations.

\subsection{Governing equations for suspended particles}

The suspended particles used in this study were assumed to be rigid, spherical, chemically stable, and non-Brownian. Their movements were simply described by Newton's second law of motion and equation of angular motion:

$$
\begin{aligned}
& \boldsymbol{F}_{\mathrm{p}}=\rho \frac{d^{2} x_{\mathrm{p}}}{d t^{2}} \\
& T_{\mathrm{p}}=I \frac{d^{2} \theta_{\mathrm{p}}}{d t^{2}}
\end{aligned}
$$


where $\boldsymbol{F}_{\mathrm{p}}$ is the external hydrodynamic force vector acting on the particle, $\rho$ is the density, $\boldsymbol{x}_{\mathrm{p}}$ is the position vector, $T_{\mathrm{p}}$ is the torque, $I$ is the moment of inertia, and $\theta_{\mathrm{p}}$ is the angle of the particle. Note that both densities of suspended particles and suspending fluid were assumed to be equivalent for neutral buoyancy. The external force vector $\boldsymbol{F}_{\mathrm{p}}$ and torque $T_{\mathrm{p}}$ acting on the particles were discretized by a third-order Adams-Bashforth method and solved numerically by a two-way coupling scheme [7]:

$$
\begin{gathered}
\dot{x}_{\mathrm{p}}^{n+1}=\dot{x}_{\mathrm{p}}^{n}+\Delta t \frac{23 F_{\mathrm{p}}^{n}-16 F_{\mathrm{p}}^{n-1}+5 F_{\mathrm{p}}^{n-2}}{12 \rho}, \\
\boldsymbol{x}_{\mathrm{p}}^{n+1}=x_{\mathrm{p}}^{n}+\Delta t \frac{5 \dot{x}_{\mathrm{p}}^{n+1}+8 \dot{x}_{\mathrm{p}}^{n}-\dot{x}_{\mathrm{p}}^{n-1}}{12}, \\
\dot{\theta}_{\mathrm{p}}^{n+1}=\dot{\theta}_{\mathrm{p}}^{n}+\Delta t \frac{23 T_{\mathrm{p}}^{n}-16 T_{\mathrm{p}}^{n-1}+5 T_{\mathrm{p}}^{n-2}}{12 I}, \\
\theta_{\mathrm{p}}^{n+1}=\theta_{\mathrm{p}}^{n}+\Delta t \frac{5 \dot{\theta}_{\mathrm{p}}^{n+1}+8 \dot{\theta}_{\mathrm{p}}^{n}-\dot{\theta}_{\mathrm{p}}^{n-1}}{12} .
\end{gathered}
$$

\section{RESULTS AND DISCUSSION}

The macroscopic axial velocity and microscopic particles behavior at $t=8 \mathrm{~s}$ are depicted in Fig. 2. The suspended particles are shown to flow randomly in a channel. Note that since sufficient number of grids was allocated in the computational domain, collisions between particles or particle and channel wall were not observed. At least 2 or 3 grids always remained between the particles during the simulations. Since inertial effects were negligible due to low Reynolds number condition, the particles did not migrate in the width direction, i.e., the particles flowed almost along the macroscopic streamline. The particles, however, did not flow very near the channel wall. The particles apparently flowed avoiding a certain peripheral layer near the channel wall in Fig. 2(a) and 2(b). This is because repulsive force from the wall increased exponentially with approaching the wall [6], [14]. Owing to these effects, the particles did not approach the wall and some layers without particles existed near the wall. However, for the case $\phi=4.07 \%$, particles flowed within these regions too as shown in Fig. 2(c). This is partly because when particles are getting jammed with increasing concentration, some particles are pushed away toward the peripheral layers near the wall. These phenomena are sometimes observed in microcirculation termed "margination". Deformable red blood cells often exhibit axial migration, and leukocytes appear to flow primarily in the peripheral layer to the contrary, which is the first step in the firm adhesion to the endothelium [20]. In this study, the suspended particles were rigid and their shape and size were all the same. Judging from our results, it might be stated that margination occurs depending on concentration of the particles, when the particles are all rigid and spherical.

To consider the velocity profiles composed of the particles, the $y$-axis position and axial velocity of the particles were plotted in Fig. 3. The solid line indicates that from the Newtonian fluid. Our numerical data were in good agreement with that from the Newtonian parabolic fluid. This is because particles flowed in accordance with the streamlines of the macroscopic flows due to sufficiently weak inertial forces. The particles were scattered almost uniformly along the width ( $y$-axis) direction, except for the peripheral layers near the wall $(y / l= \pm 1.0)$ as mentioned above. However, some particles were pushed away toward the wall side and flowed within the peripheral layers for the case $\phi=4.07 \%$. 

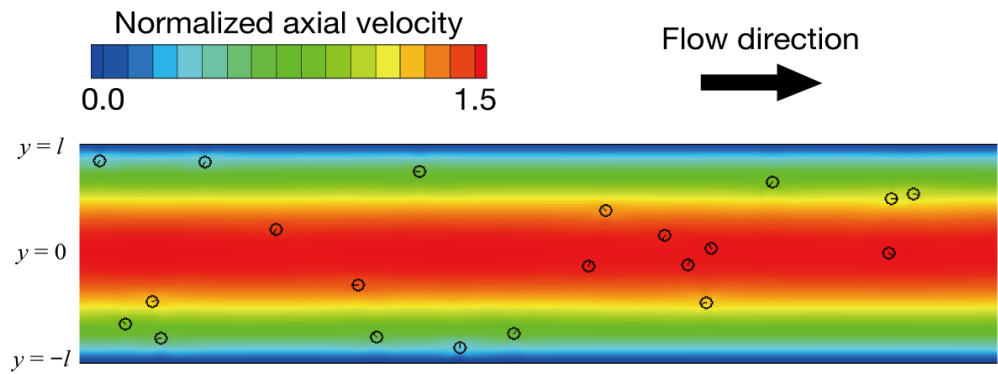

(a) concentration $\phi=1.02 \%$

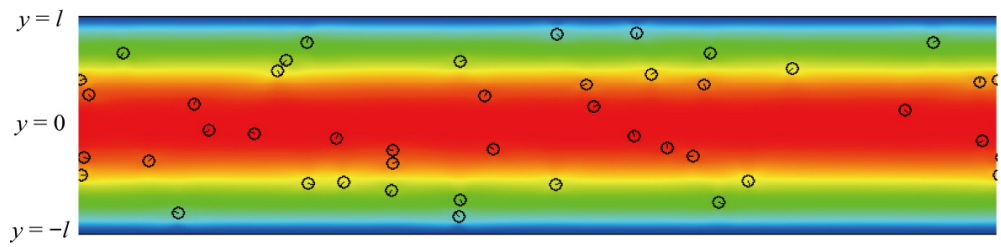

(b) concentration $\phi=2.04 \%$

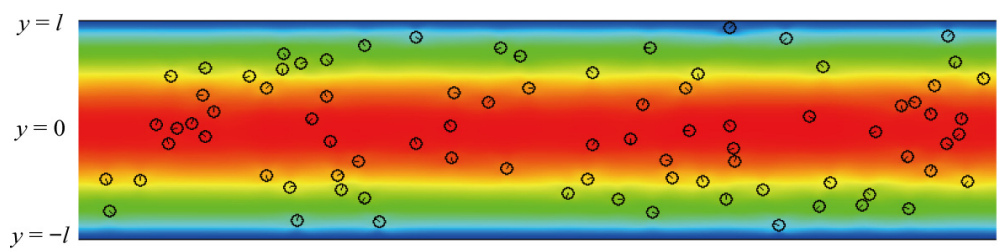

(c) concentration $\phi=4.07 \%$

Figure 2: Snapshots of pressure-driven suspension flows at time $t=8 \mathrm{~s}$. (a) concentration $\phi=1.02 \%$; (b) concentration $\phi=2.04 \%$; and (c) concentration $\phi=4.07 \%$, respectively.

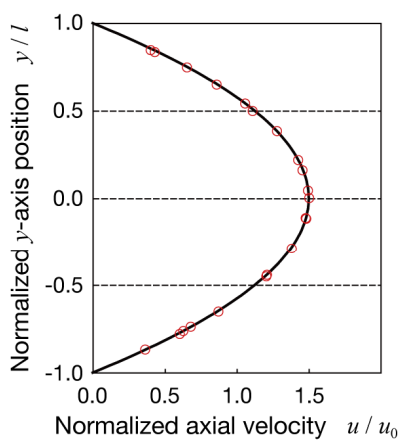

(a) concentration $\phi=1.02 \%$

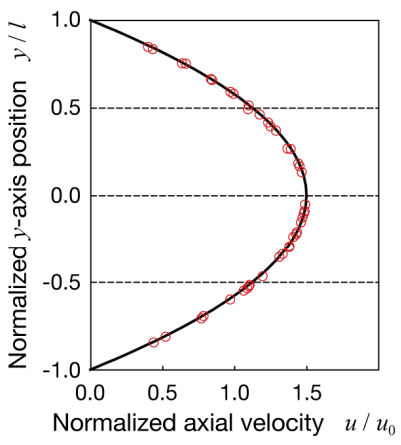

(b) concentration $\phi=2.04 \%$

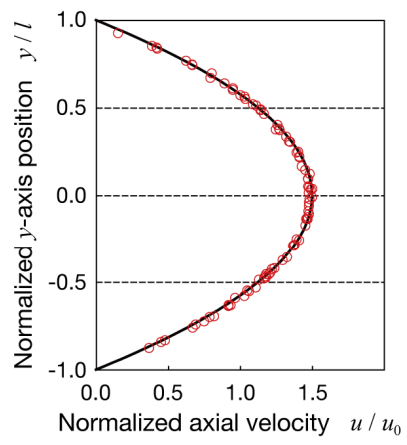

(c) concentration $\phi=4.07 \%$

Figure 3: Axial velocity profile composed of the suspended particles. The solid line indicates that from the Newtonian fluid: (a) concentration $\phi=1.02 \%$; (b) concentration $\phi=2.04 \%$; and (c) concentration $\phi=4.07 \%$, respectively. 
Number of particles versus $y$-axis position was obtained as a function of probability density function (PDF) to consider their dispersed states. Relationship between PDF and $y$ axis position is also useful to consider its microstructure, i.e., spatial arrangement of the suspended particles. In this study, $y$-axis position was divided into 20 segments as shown in Fig. 4. Therefore, when the particles are homogeneously dispersed, the PDF value corresponds to $1 / 20=0.05$ as denoted by solid line in Fig. 4 . Note that the value of 0.05 also corresponds to the confinement, i.e., ratio of particle size to the channel width. The data are plotted together with the standard error (SE) of the mean. Each SE was sufficiently small, which indicates number of trials in order to exclude initial position effects would be plenty. It was found that the PDF values were around 0.05 with some variations except for the peripheral layers $(y / l= \pm 1.0)$, indicating the particles flowed homogeneously and were dispersed uniformly in the $y$-axis direction. This is because inertial effects of the particles were negligible for low Reynolds number condition as we discussed in previous studies [8], [15], [21]. It is worth mentioning that the values of PDF were almost zero, i.e., no particles were observed, in the peripheral layers for the case $\phi=1.02$ and $2.04 \%$ due to strong repulsive forces from the channel wall. For the case $\phi=4.07 \%$, on the other hand, they were more flat around 0.05 including the peripheral layers as discussed above. Fig. 4 clearly shows differences in the dispersed states of the particles due to margination. Accordingly, microstructure of suspension can be easily visualized, compared and considered by using PDF.

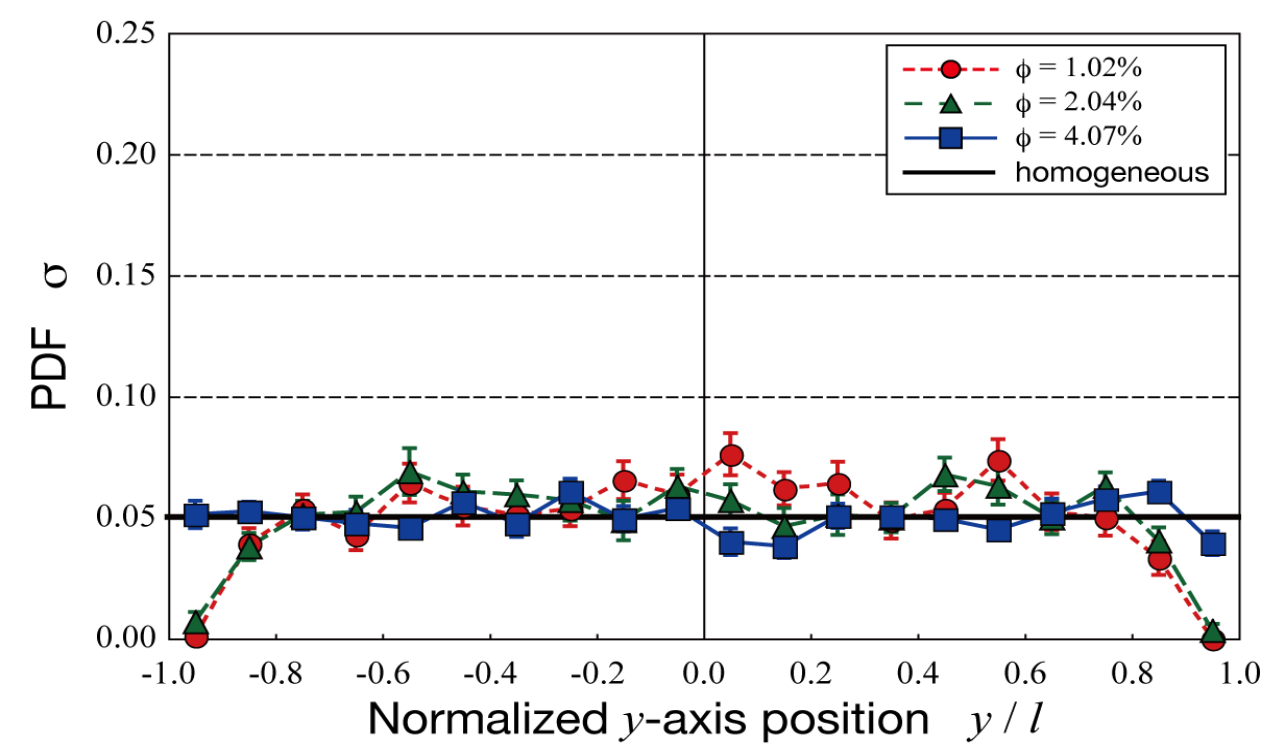

Figure 4: Relationship between probability density function (PDF) and normalized $y$-axis position. The data are plotted together with \pm 1 SE. The solid line denotes homogeneously dispersed state in the $y$ axial direction.

Relative viscosity for each concentration is shown in Fig. 5. Data plotted are mean \pm 1 standard error (SE). The error bars were sufficiently small, which indicates effects of initial particles' positions were properly removed. The solid line denotes that form Einstein's viscosity equation [2]. Our results were in good agreement with the theoretical values for 
lower concentration conditions, which indicates our computational conditions satisfied some assumptions in Einstein's equation, i.e., sufficiently small particles, negligibly weak inertial forces, homogeneously dispersed state, and low concentration. On the other hand, our data for $\phi=4.07 \%$ significantly differed from that by Einstein. Our data corresponded to that for $\phi=6.8 \%$ by Einstein's equation, which is 1.7 times as high as the actual concentration. We previously considered one of the reasons for nonlinearly enhanced relative viscosity for higher concentration suspensions from the viewpoint of rotational motions of the suspended particles due to hydrodynamic interactions [7]. In addition to this consideration, present study takes account of microstructure of the suspended particles. For higher concentration suspensions, particles flowed within the peripheral layers due to margination. When the macroscopic total effective viscosity can be discussed by summation of each microscopic particle's contribution to the effective viscosity, particles near the channel wall yield major and significant contributions [6], [14]. Then the intrinsic viscosity, which corresponds to a ratio of viscosity to concentration, was consequently increased with concentration. Microstructure using PDF would be a promising index to consider rheological properties of a suspension.

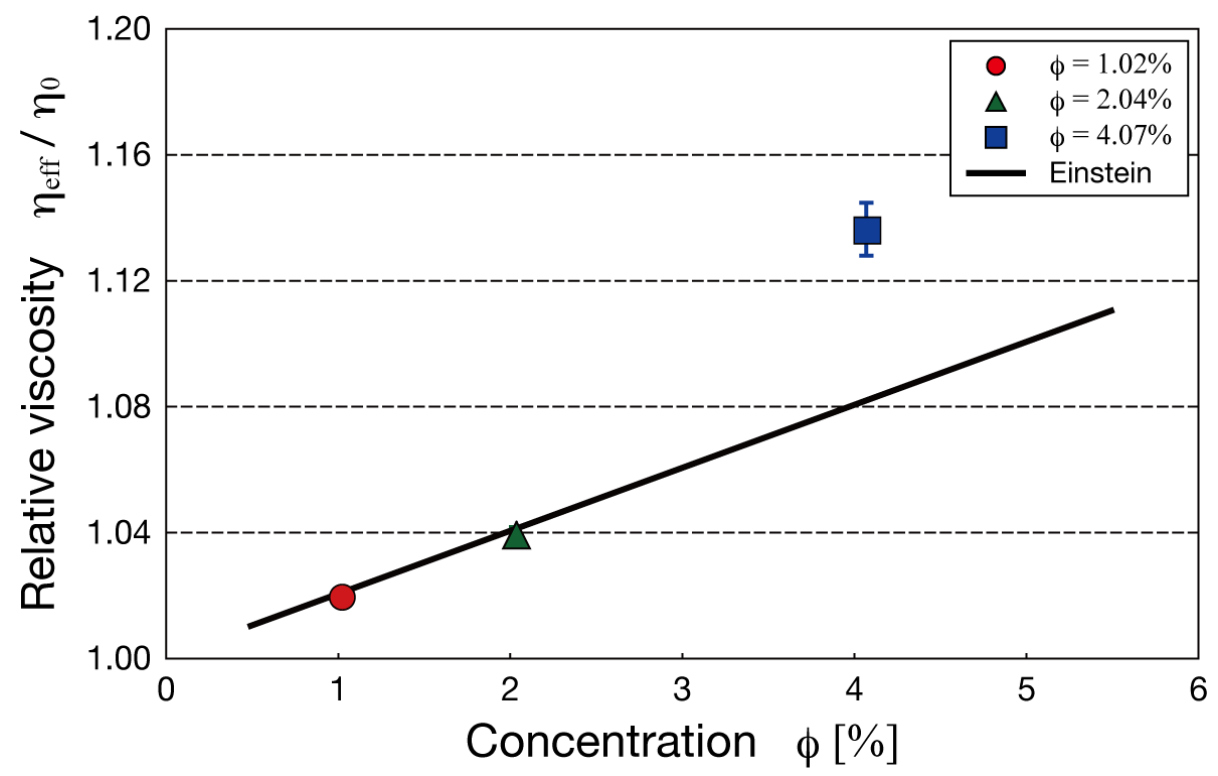

Figure 5: Relationship between relative viscosity and concentration of a particle suspension. The data are plotted together with \pm 1 SE. The solid line denotes that from Einstein's viscosity equation.

\section{CONCLUSIONS}

Two-dimensional pressure-driven suspension flow simulations were conducted by a twoway coupling scheme in order to consider relationship between microstructure and consequent relative and intrinsic viscosities. As a result, for the case $\phi=4.07 \%$, the particles were dispersed homogeneously including peripheral layers due to margination. Then the relative and intrinsic viscosities were consequently enhanced and exceeded those from Einstein's estimation. Since the intrinsic viscosity was significantly related to the 
microstructure of the suspension, microstructure using PDF would be a promising index to consider rheological properties of a suspension.

\section{ACKNOWLEDGEMENT}

This work was supported in part by JSPS KAKENHI Grant Number JP20K04266.

\section{REFERENCES}

[1] Einstein, A., Eine neue bestimmung der molekuldimensionen. Annals of Physics, 19, pp. 289-306, 1906.

[2] Brady, J.F., The Einstein viscosity correction in n dimensions. International Journal of Multiphase Flow, 10, pp. 113-114, 1984.

[3] Thomas, D.G., Transport characteristics of suspension: 8. A note on the viscosity of Newtonian suspensions of uniform spherical particles. Journal of Colloid Science, 20, pp. 267-277, 1965.

[4] Ghigliotti, G., Biben, T. \& Misbah, C., Rheology of a dilute two-dimensional suspension of vesicles. Journal of Fluid Mechanics, 653, pp. 489-518, 2010.

[5] Mueller, S., Llewellin, E.W. \& Mader, H.M., The rheology of suspension of solid particles. Proceedings of the Royal Society A, 466, pp. 1201-1228, 2010.

[6] Doyeux, V., Priem, S., Jibuti, L. \& Farutin, A., Effective viscosity of twodimensional suspensions: Confinement effects. Physical Review Fluids, 1, 043301, pp. 1-22, 2016.

[7] Fukui, T., Kawaguchi, M. \& Morinishi, K., A two-way coupling scheme to model the effects of particle rotation on the rheological properties of a semidilute suspension. Computers \& Fluids, 173, pp. 6-16, 2018.

[8] Fukui, T., Kawaguchi, M. \& Morinishi, K., Numerical study on the inertial effects of particles on the rheology of a suspension. Advances in Mechanical Engineering, 11, pp. 1-10, 2019.

[9] Hu, X., Lin, J. \& Ku, X., Inertial migration of circular particles in Poiseuille flow of a power-law fluid. Physics of Fluids, 31, 073306, pp. 1-15, 2019.

[10] Christ, F.E., Bowie, S. \& Alexeev, A., Inertial migration of spherical particles in channel flow of power law fluids. Physics of Fluids, 32, 083103, pp. 1-8, 2020.

[11] Tanaka, M., Fukui, T., Kawaguchi, M. \& Morinishi, K., Numerical simulation on the effects of power-law fluidic properties on the suspension rheology. Journal of Fluid Science and Technology, to be published.

[12] Melander, O., Modrego, J., Zamorano-León, J.J., Santos-Sancho, J.M., Lahera, V. \& López-Farré, A.J., New circulating biomarkers for predicting cardiovascular death in healthy population. Journal of Cellular and Molecular Medicine, 19, pp. 2489-2499, 2015.

[13] Stickel, J.J. \& Powell, R.L., Fluid mechanics and rheology of dense suspensions. Annual Review of Fluid Mechanics, 37, pp. 129-149, 2005.

[14] Okamura, N., Fukui, T., Kawaguchi, M. \& Morinishi, K., Influence of each cylinder's contribution on the total effective viscosity of a two-dimensional suspension by a two-way coupling scheme. Journal of Fluid Science and Technology, to be published.

[15] Fukui, T., Kawaguchi, M. \& Morinishi, K., Numerical study of the microstructure of a dilute suspension to assess its thixotropic behavior by a two-way coupling scheme. WIT Transactions on Engineering Sciences, vol. 128, WIT Press: Southampton and Boston, pp. 47-58, 2020. 
100 Advances in Fluid Dynamics with emphasis on Multiphase and Complex Flow

[16] Morinishi, K. \& Fukui, T., An Eulerian approach for fluid-structure interaction problems. Computers \& Fluids, 65, pp. 92-98, 2012.

[17] Izham, M., Fukui, T. \& Morinishi, K., Application of regularized lattice Boltzmann method for incompressible flow simulation at high Reynolds number and flow with curved boundary. Journal of Fluid Science and Technology, 6, pp. 812-821, 2011.

[18] Morinishi, K. \& Fukui, T., Parallel computation of turbulent flows using moment base lattice Boltzmann method. International Journal of Computational Fluid Dynamics, 30, pp. 363-369, 2016.

[19] Sterling, J.D. \& Chen, S., Stability analysis of lattice Boltzmann methods. Journal of Computational Physics, 123, pp. 196-206, 1996.

[20] Takeishi, N., Imai, Y., Nakaaki, K., Yamaguchi, T. \& Ishikawa, T., Leukocyte margination at arteriole shear rate. Physiological Reports, 2, pp. 1-8, e12037, 2014.

[21] Kawaguchi, M. et al., Viscosity estimation of a suspension with rigid spheres in circular microchannels using particle tracking velocimetry. Micromachines, 10(10), 675, pp. 1-13, 2019. 